\title{
The timescale of low-mass proto-helium white dwarf evolution ${ }^{\star}$
}

\author{
A. G. Istrate ${ }^{1}$, T. M. Tauris ${ }^{1,2}$, N. Langer ${ }^{1}$, and J. Antoniadis ${ }^{2}$ \\ 1 Argelander-Institut für Astronomie, Universität Bonn, Auf dem Hügel 71, 53121 Bonn, Germany \\ e-mail: aistrate@astro.uni-bonn.de \\ 2 Max-Planck-Institut für Radioastronomie, Auf dem Hügel 69, 53121 Bonn, Germany
}

Received 25 July 2014 / Accepted 17 October 2014

\begin{abstract}
Context. A large number of low-mass $\left(\$ 0.20 M_{\odot}\right)$ helium white dwarfs (He WDs) have recently been discovered. The majority of these are orbiting another WD or a millisecond pulsar (MSP) in a close binary system; a few examples are found to show pulsations or to have a main-sequence star companion. There appear to be discrepancies between the current theoretical modelling of such lowmass He WDs and a number of key observed cases, indicating that their formation scenario yet remains to be fully understood.

Aims. Here we investigate the formation of detached proto-He WDs in close-orbit low-mass X-ray binaries (LMXBs). Our prime focus is to examine the thermal evolution and the contraction phase towards the WD cooling track and investigate how this evolution depends on the WD mass. Our calculations are then compared to the most recent observational data.

Methods. Numerical calculations with a detailed stellar evolution code were used to trace the mass-transfer phase in a large number of close-orbit LMXBs with different initial values of donor star mass, neutron star mass, orbital period, and strength of magnetic braking. Subsequently, we followed the evolution of the detached low-mass proto-He WDs, including stages with residual shell hydrogen burning and vigorous flashes caused by unstable CNO burning.

Results. We find that the time between Roche-lobe detachment until the low-mass proto-He WD reaches the WD cooling track is typically $\Delta t_{\text {proto }}=0.5-2 \mathrm{Gyr}$, depending systematically on the WD mass and therefore on its luminosity. The lowest WD mass for developing shell flashes is $\sim 0.21 M_{\odot}$ for progenitor stars of mass $M_{2} \leq 1.5 M_{\odot}$ (and $\sim 0.18 M_{\odot}$ for $M_{2}=1.6 M_{\odot}$ ).

Conclusions. The long timescale of low-mass proto-He WD evolution can explain a number of recent observations, including some MSP systems hosting He WD companions with very low surface gravities and high effective temperatures. We find no evidence for $\Delta t_{\text {proto }}$ to depend on the occurrence of flashes and thus question the suggested dichotomy in thermal evolution of proto-WDs.
\end{abstract}

Key words. white dwarfs - stars: evolution - binaries: close - X-rays: binaries - stars: neutron

\section{Introduction}

In recent years, the number of detected low-mass $\left(\lessgtr 0.20 M_{\odot}\right)$ helium white dwarfs (He WDs) has increased dramatically, mainly as a result of multiple survey campaigns such as WASP, ELM, HVS, Kepler, and SDSS (Pollacco et al. 2006; Rowe et al. 2010; Brown et al. 2005, 2010, 2013; Silvotti et al. 2012; Kilic et al. 2012; Hermes et al. 2013; Maxted et al. 2014).

The existence of low-mass He WDs in close binaries with a radio millisecond pulsar (MSP), however, has been known for a few decades (e.g. van Kerkwijk et al. 2005, and references therein). Several attempts have been made to calibrate WD cooling models for such systems on the basis of the spin-down properties of the MSP (e.g. Alberts et al. 1996; Hansen \& Phinney 1998; Driebe et al. 1998; Althaus et al. 2001; Panei et al. 2007). The idea is that the characteristic spin-down age of the MSP $\left(\tau_{\mathrm{PSR}} \equiv P /(2 \dot{P})\right.$, where $P$ is the spin period and $\dot{P}$ is the period derivative) should be equivalent to the cooling age of the WD $\left(\tau_{\text {cool }}\right)$, assuming that the radio MSP is activated at the same time as the WD is formed, following an epoch of mass transfer in a low-mass X-ray binary (LMXB). Unfortunately, this method is highly problematic since $\tau_{\text {PSR }}$ generally is a poor true age estimator. It can easily be incorrect by a factor of 10 or more (Camilo et al. 1994; Lorimer et al. 1995; Tauris 2012; Tauris et al. 2012). Determining the true age of MSPs, however, is

* Appendices are available in electronic form at http://www . aanda.org important for studying their spin evolution and constraining the physics of their previous recycling phase (Lazarus et al. 2014).

The discovery of the intriguing PSR J1012+5307 (Nicastro et al. 1995) sparked an intense discussion about WD cooling ages and MSP birthrates (Lorimer et al. 1995) given that $\tau_{\text {PSR }}>20 \tau_{\text {cool }}$. Soon thereafter, it was suggested (Alberts et al. 1996; Driebe et al. 1998; van Kerkwijk et al. 2005) that He WDs with a mass $\$ 0.20 M_{\odot}$ avoid hydrogen shell flashes, whereby their relatively thick $\left(\sim 10^{-2} M_{\odot}\right)$ hydrogen envelope remains intact, causing residual hydrogen shell burning to continue on a very long timescale. Despite significant theoretical progress (e.g. Althaus et al. 2013, and references therein), our understanding of the thermal evolution of (proto) He WDs remains uncertain. In particular, a number of recent observations of apparently bloated WDs calls for an explanation.

In this Letter, we study the formation of a large number of low-mass He WDs by modelling close-orbit LMXBs. We carefully investigate the properties of the resulting protoWDs and follow their evolution until and beyond settling on the WD cooling track. Finally, we compare our results with observations.

\section{Numerical methods and physical assumptions}

Numerical calculations with a detailed stellar evolution code were used to trace the mass-transfer phase following the same prescription as outlined in Istrate et al. (2014). We investigated models with a metallicity of $Z=0.02$, a mixing-length 


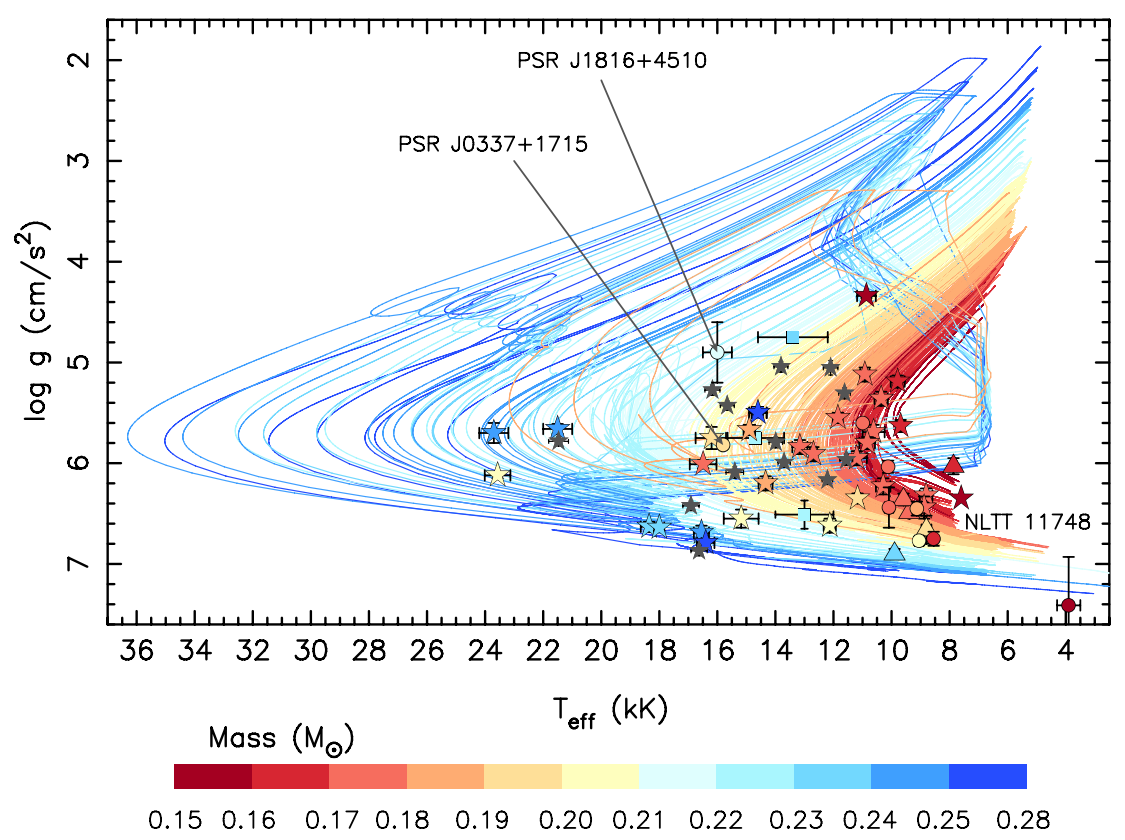

Fig. 1. Evolutionary tracks in the $\left(T_{\text {eff }}, \log g\right)$-diagram. The evolution from Roche-lobe detachment until settling on the WD cooling track and beyond is shown for a selection of our models. The colour scale represents the final WD mass. A few cases of vigorous hydrogen shell flashes explain the large (counterclockwise) loops in the diagram. Observed WDs are shown with symbols (stars: sdB+WD, double WDs (grey stars: WDs with poor mass constraints); triangles: pulsating WDs; squares: WD+MS; circles: WD+MSP - see Appendix A for references and data).

parameter $\alpha=l / H_{\mathrm{p}}=2.0$, and a core convective overshooting parameter of $\delta_{\mathrm{OV}}=0.10$. A wide range of LMXB systems were investigated with different initial values of donor star mass $\left(M_{2}\right)$, neutron star mass, orbital period, and the so-called $\gamma$-index of magnetic braking. The evolution of the low-mass (proto) He WD was calculated including chemical diffusion (mixing), hydrogen shell flashes (CNO burning), and residual shell hydrogen burning. Convective, semi-convective, and overshoot mixing processes were treated via diffusion. Thermohaline mixing was included as well, whereas gravitational settling and radiative levitation were neglected, as was stellar wind mass loss.

\section{Results}

In Fig. 1 we have plotted a selection of our calculated evolutionary tracks, from the moment of Roche-lobe detachment until the end of our calculations, for (proto) He WDs with masses of $0.15-0.28 M_{\odot}$. In general, our models fit the observations quite well. The few cases with discrepancies are sources with large uncertainties in the WD mass. Vigorous single or multiple cycle hydrogen shell flashes explain the large loops in the diagram, whereas mild thermal instabilities are seen e.g. for the $0.25 M_{\odot}$ proto-WDs at $\log g \simeq 4.5$. It has been known for many years that a thermal runaway flash may develop through unstable CNO burning when a proto-WD evolves towards the cooling track (Kippenhahn \& Weigert 1967; Webbink 1975; Iben \& Tutukov 1986). During these flashes the luminosity becomes very high, whereby the rate of hydrogen burning is significantly increased (e.g. Nelson et al. 2004; Gautschy 2013, and references therein). Our models with strong flashes often experience an additional episode of mass loss via Roche-lobe overflow (RLO, see also Iben \& Tutukov 1986; Sarna et al. 2000; Podsiadlowski et al. 2002; Nelson et al. 2004).

For progenitor stars with $M_{2} \leq 1.5 M_{\odot}$ we find hydrogen shell flashes in WDs with masses of $0.21 \leq$ $M_{\mathrm{WD}} / M_{\odot} \leq 0.28$. Hence, the lowest mass for which flashes

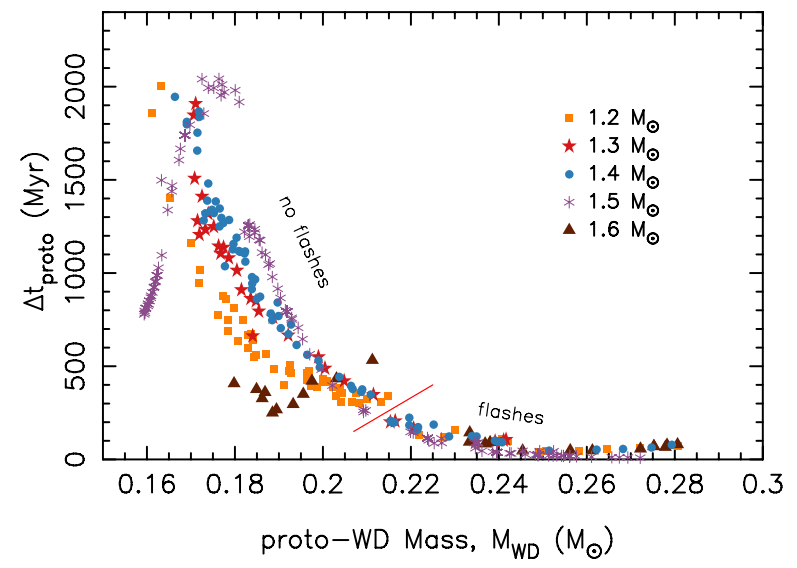

Fig. 2. Contraction timescale, $\Delta t_{\text {proto }}$ of evolution from Roche-lobe detachment until settling on the WD cooling track, plotted as a function of WD mass, $M_{\mathrm{WD}}$. The initial ZAMS masses of the WD progenitors (the LMXB donor stars) are indicated with various symbols and colours. The red line marks $M_{\text {flash }} \simeq 0.21 M_{\odot}$ for progenitor stars $\lesssim 1.5 M_{\odot}$.

occur is $M_{\text {flash }}=0.21 M_{\odot}$. However, we find a lower value of $M_{\text {flash }}=0.18 M_{\odot}$ for $M_{2}=1.6 M_{\odot}$. It has been argued (e.g. van Kerkwijk et al. 2005) that the value of $M_{\text {flash }}$ is important since it marks a dichotomy for the subsequent WD cooling such that WDs with a mass $M_{\mathrm{WD}}<M_{\text {flash }}$ remain hot on a Gyr timescale as a result of continued residual hydrogen shell burning, whereas WDs with $M_{\mathrm{WD}}>M_{\text {flash }}$ cool relatively fast as a result of the shell flashes that erode the hydrogen envelope. We find that this transition is smooth, however, and that the thermal evolution timescale mainly depends on the proto-He WD luminosity and not on the occurrence or absence of flashes.

In Fig. 2 we have plotted the time, $\Delta t_{\text {proto }}$ it takes from Roche-lobe detachment until the star reaches its highest value of $T_{\text {eff }}$. (For WDs that undergo hydrogen shell flashes we used the time until the occurrence of highest $T_{\text {eff }}$ on their last loop 
A. G. Istrate et al.: The timescale of low-mass proto-He WD evolution

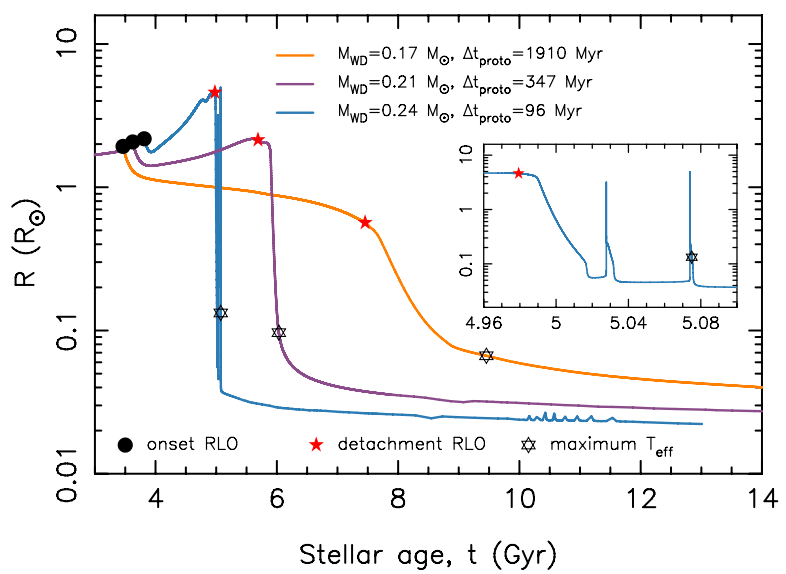

Fig. 3. Radius as a function of stellar age for the progenitor stars of three He WDs of mass $0.17,0.21$ and $0.24 M_{\odot}$. The most massive proto-WD evolves with hydrogen shell flashes - see inset. The epoch between the solid red star (RLO termination) and the open black star (max. $T_{\text {eff }}$ ) marks the contraction (transition) timescale, $\Delta t_{\text {proto }}=96-1910 \mathrm{Myr}$.

in the HR-diagram.) The plot shows a very strong dependence on $M_{\mathrm{WD}}$. For very low-mass He WDs (i.e. those with $M_{\mathrm{WD}}<$ $M_{\text {flash }}$, which therefore avoid hydrogen shell flashes), $\Delta t_{\text {proto }}$ may last up to 2 Gyr. This result has important consequences for their thermal evolution and contraction (see below). There is a well-known correlation between the degenerate core mass of an evolved low-mass star and its luminosity, $L$ (Refsdal \& Weigert 1971). After terminating the RLO, the star moves to the far left in the HR-diagram - (initially) roughly at constant $L$ - while burning the residual hydrogen in the envelope at a rate proportional to $L$. We find that the total amount of hydrogen left in the envelope is always $\sim 0.01 \pm 0.005 M_{\odot}$, in agreement with Sarna et al. (2000), and is correlated in a variable manner with $M_{\mathrm{WD}}$ (especially for $M_{2} \geq 1.5 M_{\odot}$, explaining the peak in Fig. 2). Therefore, the increase in $\Delta t_{\text {proto }}$ seen in Fig. 2 for decreasing values of $M_{\mathrm{WD}}$ can simply be understood from their much lower luminosities following the Roche-lobe detachment (see also Figs. 5 and 10 in Istrate et al. 2014). Based on our calculated proto-He WD models, we find (see Appendix B)

$\Delta t_{\text {proto }} \simeq 400 \mathrm{Myr}\left(\frac{0.20 M_{\odot}}{M_{\mathrm{WD}}}\right)^{7}$.

The conclusion that $\Delta t_{\text {proto }}$ can reach $\sim$ Gyr was found previously for a few single models (e.g. Driebe et al. 1998; Sarna et al. 2000; Althaus et al. 2001). Here we show, for the first time, its systematic dependence on $M_{\mathrm{WD}}$.

Figure 3 shows the contraction phase for three proto-He WDs. The value of $\Delta t_{\text {proto }}$ increases significantly when $M_{\mathrm{WD}}$ decreases from 0.24 to $0.17 M_{\odot}$. Hence, low-mass $\left(\lesssim 0.20 M_{\odot}\right)$ proto-He WDs can remain bloated on a very long timescale. It is important to notice that no pronounced discontinuity in $\Delta t_{\text {proto }}$ is seen at $M_{\text {flash }} \simeq 0.21 M_{\odot}$ (cf. Figs. 2,3 , and C.1). Although the peak luminosity (and thus the rate of eroding hydrogen) is high during a flash, the star only spends a relatively short time $\left(\sim 10^{3}-10^{6} \mathrm{yr}\right)$ at high $L$ when making a loop in the HR-diagram.

\section{Comparison with observational data of He WDs}

In Table A.1 we list observed low-mass He WDs included among the plotted data in Fig. 1. We now discuss recent interesting sources in view of our theoretical modelling.

\subsection{MSPs with low-mass (proto) He WDs in tight orbits}

The companion star to PSR J1816+4510 $\left(P_{\text {orb }}=8.7 \mathrm{~h}\right)$ was recently observed by Kaplan et al. (2012, 2013). They assembled optical spectroscopy and found an effective temperature of $T_{\text {eff }}=16000 \pm 500 \mathrm{~K}$, a surface gravity of $\log g=4.9 \pm 0.3$, and a companion mass of $M_{\mathrm{WD}} \sin ^{3} i=0.193 \pm 0.012 M_{\odot}$, where $i$ is the orbital inclination angle of the binary. They concluded that while the spectrum is rather similar to that of a lowmass $\mathrm{He} \mathrm{WD}$, it has a much lower surface gravity (i.e. larger radius) than a WD on the cooling track. They discussed that PSR J1816+4510 perhaps represents a redback system (cf. Chen et al. 2013, for a formation scenario) where pulsar irradiation of the hydrogen-rich, bloated companion causes evaporation of material, which can explain the observed eclipses of the radio pulses for $\sim 10 \%$ of the orbit. However, the very hot surface temperature of this companion $(16000 \mathrm{~K})$ cannot be explained from a redback scenario. Redbacks typically have illuminated dayside temperatures of only $T_{\text {eff }} \simeq 6000 \mathrm{~K}$ (Breton et al. 2013). Here we suggest that this companion star is simply a low-mass proto-He WD. As we have demonstrated, such a star takes several $100 \mathrm{Myr}$ to reach the cooling track, and our models match the observed values of $T_{\text {eff }}$ and $\log g$. (Note, for $P_{\text {orb }}=8.7 \mathrm{~h}$ one usually expects $M_{\mathrm{WD}} \lesssim 0.18 M_{\odot}$, cf. Istrate et al. 2014.)

Another case is the triple system PSR J0337+1715 recently discovered by Ransom et al. (2014), which raises fundamental questions about its formation (Tauris \& van den Heuvel 2014). One open question is the order of formation of the two WDs orbiting the MSP (with $P_{\text {orb }}=1.6$ and 327 days). Spectroscopy of the inner companion by Kaplan et al. (2014b) verified that this is a $0.197 M_{\odot} \mathrm{He} \mathrm{WD}$, as known from pulsar timing. They measured a low surface gravity of $\log g=5.82 \pm 0.05$ and noted that its very high surface temperature, $T_{\text {eff }}=15800 \pm 100 \mathrm{~K}$, could indicate that it had just experienced a flash. This would suggest a surprisingly short lifetime for this object. However, our modelling of $\sim 0.20 M_{\odot}$ He WDs shows that these stars avoid flashes. Instead we find that for such a star it takes 400-600 Myr (Fig. 2) to reach the WD cooling track. Therefore, we conclude that it is reasonable to detect such a WD at an early, bloated stage of its evolution.

\subsection{NLTT 11748 and other low-mass (proto) He WD binaries}

A large number of low-mass proto-He WDs (also classified as sdB stars) are found in binaries with another WD. These systems probably formed via stable RLO in cataclysmic variable systems resembling our calculations, but with a $\sim 0.7 M_{\odot}$ CO WD accretor instead of a NS. NLTT 11748 was discovered by Steinfadt et al. (2010), with follow-up observations made by Kaplan et al. (2014a). This eclipsing detached binary consists of a $0.71-0.74 M_{\odot}$ CO WD with a very low-mass He WD and $P_{\text {orb }} \simeq 5.6 \mathrm{~h}$. Our evolutionary tracks for a $0.16 M_{\odot}$ He WD are indeed consistent with their observed values of $\log g=6.35$ and $T_{\text {eff }}=7600 \mathrm{~K}$ (and their estimated mass of 0.136-0.162 $M_{\odot}$ ). Brown et al. (2013) recently detected four binaries with lowmass WDs having $\log g \simeq 5$, in accordance with our modelling of proto-He WDs presented here (cf. Figs. 1 and A.1).

\subsection{Bloated, hot, low-mass He WDs detected by Kepler}

Four (proto) He WDs have been found with A-star companions in the combined transit and eclipse data from the Kepler mission (van Kerkwijk et al. 2010; Carter et al. 2011; Breton et al. 2012). Three of these He WDs (KOI-74, KIC 10657664, 
KOI-1224) have $M_{\mathrm{WD}} \lesssim 0.26 M_{\odot}$ and are also plotted in Fig. 1. The mass estimates of these WDs are not very precise. However, within $1-2 \sigma$, the characteristics of these objects also seem to match our evolutionary tracks reasonably well.

The question now is why we see all these bloated proto-WDs given that WDs spend significantly more time on the subsequent cooling tracks. This is simply a selection effect. The WDs are only seen to eclipse A-stars in the Kepler data as long as they are bloated proto-WDs (and thus also more luminous than ordinary WDs, which have already settled on the cooling track).

\section{Discussion and conclusions}

We have demonstrated that low-mass $\left(\$ 0.20 M_{\odot}\right)$, detached proto-He WDs may spend up to $\sim 2 \mathrm{Gyr}$ in the contraction (transition) phase from the Roche-lobe detachment until they reach the WD cooling track. This is important for an age determination of He WDs in general and for recycled MSPs in particular. We expect a fair number of He WDs to be observed in this (bloated) phase, in agreement with recent observations.

The duration of this contraction phase $\left(\Delta t_{\text {proto }}\right)$ decreases strongly with increasing mass of the proto-He WD, $M_{\mathrm{WD}}$. This can be understood from the well-known correlation between degenerate core mass and luminosity of an evolved low-mass star. Therefore, after Roche-lobe detachment, the rate at which the residual $\left(0.01 \pm 0.005 M_{\odot}\right)$ hydrogen in the envelope is consumed is directly proportional to the luminosity and thus $M_{\mathrm{WD}}$. The value of $\Delta t_{\text {proto }}$ is not particularly sensitive to the occurrence or absence of flashes.

Whether or not hydrogen shell flashes occur depends on the WD mass, its chemical composition, and the treatment of diffusion (mixing) of the chemical elements (e.g. Driebe et al. 1998; Sarna et al. 2000; Althaus et al. 2001; Nelson et al. 2004; Althaus et al. 2013). In general, we find flashes in our models with $0.21 \leq M_{\mathrm{WD}} / M_{\odot} \leq 0.28$ for $M_{2} \leq 1.5 M_{\odot}$. This result agrees excellently well agreement with the interval found by Nelson et al. (2004) for donors with solar metallicity, and also with the earlier work of Driebe et al. (1998). For $M_{2}=1.6 M_{\odot}$ WDs down to $\sim 0.18 M_{\odot}$ are experiencing flashes.

Detailed studies by Althaus et al. $(2001,2013)$ found hydrogen shell flashes for a much broader range of final WD mass $\left(0.18<M_{\mathrm{WD}} / M_{\odot}<0.41\right)$. However, as pointed out by Nelson et al. (2004), diffusion is an extremely fragile process, and turbulence can mitigate its effects. And more importantly, Nelson et al. (2004) find that both $M_{2}$ and the mode of angular momentum losses may also affect the range for which hydrogen shell flashes occur. Indeed, we found a lower value of $M_{\text {flash }}=0.18 M_{\odot}$ for our models with $M_{2}=1.6 M_{\odot}$. It has previously been shown that $M_{\text {flash }}$ strongly increases with lower metallicity (e.g. Sarna et al. 2000; Nelson et al. 2004). The work of Althaus et al. (2013) was calculated for a constant $M_{2}=1.0 M_{\odot}(Z=0.01)$. We have excluded such models with $M_{2}<1.1 M_{\odot}(Z=0.02)$ since these progenitor stars do not detach from their LMXB and evolve onto WD cooling track within a Hubble time.

Chemical diffusion via gravitational settling and radiative levitation was not included in this work. These effects seem to slightly increase $\Delta t_{\text {proto }}$ compared with models without diffusion (Nelson et al., in prep.). A systematic investigation of these and other effects on $\Delta t_{\text {proto }}$ and $M_{\text {flash }}$ will be addressed in a future work.
Acknowledgements. A.G.I. acknowledges discussions with L. Nelson, P. Marchant, R. Stancliffe and L. Grassitelli. J.A. acknowledges financial support by the ERC Starting Grant No. 279702 (BEACON, led by P. Freire).

\section{References}

Alberts, F., Savonije, G. J., van den Heuvel, E. P. J., \& Pols, O. R. 1996, Nature, 380,676

Althaus, L. G., Serenelli, A. M., \& Benvenuto, O. G. 2001, MNRAS, 324, 617 Althaus, L. G., Miller Bertolami, M. M., \& Córsico, A. H. 2013, A\&A, 557, A19

Antoniadis, J. I. 2013, Ph.D. Thesis, University of Bonn, Germany

Antoniadis, J., van Kerkwijk, M. H., Koester, D., et al. 2012, MNRAS, 423, 3316 Antoniadis, J., Freire, P. C. C., Wex, N., et al. 2013, Science, 340, 448

Bassa, C. G., van Kerkwijk, M. H., Koester, D., \& Verbunt, F. 2006a, A\&A, 456, 295

Bassa, C. G., van Kerkwijk, M. H., \& Kulkarni, S. R. 2006b, A\&A, 450, 295

Breton, R. P., Rappaport, S. A., van Kerkwijk, M. H., \& Carter, J. A. 2012, ApJ, 748, 115

Breton, R. P., van Kerkwijk, M. H., Roberts, M. S. E., et al. 2013, ApJ, 769, 108 Brown, W. R., Geller, M. J., Kenyon, S. J., \& Kurtz, M. J. 2005, ApJ, 622, L33 Brown, W. R., Kilic, M., Allende Prieto, C., \& Kenyon, S. J. 2010, ApJ, 723, 1072

Brown, W. R., Kilic, M., Allende Prieto, C., Gianninas, A., \& Kenyon, S. J. 2013, ApJ, 769, 66

Callanan, P. J., Garnavich, P. M., \& Koester, D. 1998, MNRAS, 298, 207

Camilo, F., Thorsett, S. E., \& Kulkarni, S. R. 1994, ApJ, 421, L15

Carter, J. A., Rappaport, S., \& Fabrycky, D. 2011, ApJ, 728, 139

Chen, H.-L., Chen, X., Tauris, T. M., \& Han, Z. 2013, ApJ, 775, 27

Córsico, A. H. \& Althaus, L. G. 2014, A\&A, 569, A106

Driebe, T., Schoenberner, D., Bloecker, T., \& Herwig, F. 1998, A\&A, 339, 123

Edmonds, P. D., Gilliland, R. L., Heinke, C. O., Grindlay, J. E., \& Camilo, F. 2001, ApJ, 557, L57

Gautschy, A. 2013 [arXiv: 1303.6652]

Hansen, B. M. S., \& Phinney, E. S. 1998, MNRAS, 294, 569

Hermes, J. J., Montgomery, M. H., Gianninas, A., et al. 2013, MNRAS, 436, 3573

Iben, Jr., I. \& Tutukov, A. V. 1986, ApJ, 311, 742

Istrate, A. G., Tauris, T. M., \& Langer, N. 2014, A\&A, 571, A45

Kaplan, D. L., Stovall, K., Ransom, S. M., et al. 2012, ApJ, 753, 174

Kaplan, D. L., Bhalerao, V. B., van Kerkwijk, M. H., et al. 2013, ApJ, 765, 158

Kaplan, D. L., Marsh, T. R., Walker, A. N., et al. 2014a, ApJ, 780, 167

Kaplan, D. L., van Kerkwijk, M. H., Koester, D., et al. 2014b, ApJ, 783, L23

Kilic, M., Brown, W. R., Allende Prieto, C., et al. 2012, ApJ, 751, 141

Kippenhahn, R., \& Weigert, A. 1967, Z. Astrophys., 65, 251

Lazarus, P., Tauris, T. M., Knispel, B., et al. 2014, MNRAS, 437, 1485

Lorimer, D. R., Lyne, A. G., Festin, L., \& Nicastro, L. 1995, Nature, 376, 393

Maxted, P. F. L., Bloemen, S., Heber, U., et al. 2014, MNRAS, 437, 1681

Nelson, L. A., Dubeau, E., \& MacCannell, K. A. 2004, ApJ, 616, 1124

Nicastro, L., Lyne, A. G., Lorimer, D. R., et al. 1995, MNRAS, 273, L68

Panei, J. A., Althaus, L. G., Chen, X., \& Han, Z. 2007, MNRAS, 382, 779

Podsiadlowski, P., Rappaport, S., \& Pfahl, E. D. 2002, ApJ, 565, 1107

Pollacco, D. L., Skillen, I., Collier Cameron, A., et al. 2006, PASP, 118, 1407

Ransom, S. M., Stairs, I. H., Archibald, A. M., et al. 2014, Nature, 505, 520

Refsdal, S. \& Weigert, A. 1971, A\&A, 13, 367

Rowe, J. F., Borucki, W. J., Koch, D., et al. 2010, ApJ, 713, L150

Sarna, M. J., Ergma, E., \& Gerškevitš-Antipova, J. 2000, MNRAS, 316, 84

Silvotti, R., Østensen, R. H., Bloemen, S., et al. 2012, MNRAS, 424, 1752

Steinfadt, J. D. R., Kaplan, D. L., Shporer, A., Bildsten, L., \& Howell, S. B. 2010, ApJ, 716, L146

Tauris, T. M. 2012, Science, 335, 561

Tauris, T. M. \& Savonije, G. J. 1999, A\&A, 350, 928

Tauris, T. M. \& van den Heuvel, E. P. J. 2014, ApJ, 781, L13

Tauris, T. M., Langer, N., \& Kramer, M. 2012, MNRAS, 425, 1601

van Kerkwijk, M. H., Bergeron, P., \& Kulkarni, S. R. 1996, ApJ, 467, L89

van Kerkwijk, M. H., Bassa, C. G., Jacoby, B. A., \& Jonker, P. G. 2005, in Binary

Radio Pulsars, eds. F. A. Rasio, \& I. H. Stairs, ASP Conf. Ser., 328, 357

van Kerkwijk, M. H., Rappaport, S. A., Breton, R. P., et al. 2010, ApJ, 715, 51

Webbink, R. F. 1975, MNRAS, 171, 555

Pages 5 to 6 are available in the electronic edition of the journal at http: //www . aanda. org 
A. G. Istrate et al.: The timescale of low-mass proto-He WD evolution

\section{Appendix A: Observational data and time evolution in the $\left(T_{\text {eff }}, \log g\right)$-diagram}

Table A.1. Observational data of a number of low-mass He WDs (and proto-He WDs), preferentially in tight binary systems.

\begin{tabular}{|c|c|c|c|c|c|}
\hline He WD & $\log g\left(\mathrm{~cm} \mathrm{~s}^{-2}\right)$ & $T_{\text {eff }}(\mathrm{K})$ & $M_{\mathrm{WD}}\left(M_{\odot}\right)$ & $P_{\text {orb }}(\mathrm{h})$ & Optical data \\
\hline PSR J0337+1715 & $5.82 \pm 0.05$ & $15800 \pm 100$ & $0.197 \pm 0.0002$ & 39.12 & Kaplan et al. (2014b) \\
\hline PSR J0348+0432 & $6.035 \pm 0.06$ & $10120 \pm 90$ & $0.172 \pm 0.003$ & 2.46 & Antoniadis et al. (2013) \\
\hline PSR J0751+1807 & $7.41 \pm 0.48$ & $3900 \pm 400$ & $0.138 \pm 0.0006^{a}$ & 6.31 & Bassa et al. (2006b) \\
\hline PSR J1012+5307 & $6.75 \pm 0.07$ & $8550 \pm 25$ & $0.16 \pm 0.02$ & 14.51 & van Kerkwijk et al. (1996); Callanan et al. (1998) \\
\hline PSR J1738+0333 & $6.45 \pm 0.07$ & $9130 \pm 150$ & $0.182 \pm 0.016$ & 8.52 & Antoniadis et al. (2012) \\
\hline PSR J1816+4510 & $4.9 \pm 0.3$ & $16000 \pm 500$ & $\sim 0.21 \pm 0.02^{b}$ & 8.66 & Kaplan et al. $(2012,2013)$ \\
\hline PSR J1909-3744 & $6.77 \pm 0.04$ & $9050 \pm 50$ & $0.2038 \pm 0.0022$ & 36.72 & Antoniadis (2013) \\
\hline PSR J0024-7204U ${ }^{c}$ & $\sim 5.6$ & $\sim 11000$ & $\sim 0.17$ & 10.29 & Edmonds et al. (2001) \\
\hline PSR J1911-5958A ${ }^{c}$ & $6.44 \pm 0.20$ & $10090 \pm 150$ & $0.175 \pm 0.010$ & 20.64 & Bassa et al. (2006a) \\
\hline NLTT 11748 & $6.35 \pm 0.03$ & $7600 \pm 120$ & $0.149 \pm 0.013$ & 5.64 & Kaplan et al. (2014a) \\
\hline KOI-74 & $6.51 \pm 0.14$ & $13000 \pm 1000$ & $0.22 \pm 0.03$ & 125.53 & van Kerkwijk et al. (2010) \\
\hline KOI-1224 & $5.75 \pm 0.06$ & $14700 \pm 1000$ & $0.22 \pm 0.02$ & 64.75 & Breton et al. (2012) \\
\hline KIC 10657664 & $5.50 \pm 0.02$ & $14600 \pm 300$ & $0.26 \pm 0.04^{d}$ & 78.55 & Carter et al. (2011) \\
\hline SDSS J184037.78 & $6.49 \pm 0.06$ & $9390 \pm 140$ & $\sim 0.17$ & 4.59 & Hermes et al. $(2013)^{e, f}$ \\
\hline SDSS J111215.82 & $6.36 \pm 0.06$ & $9590 \pm 140$ & $\sim 0.17$ & 4.14 & Hermes et al. $(2013)^{e, f}$ \\
\hline SDSS J151826.68 & $6.90 \pm 0.05$ & $9900 \pm 140$ & $\sim 0.23$ & 14.62 & Hermes et al. $(2013)^{e, f}$ \\
\hline $\mathrm{J} 1614$ & $6.66 \pm 0.14$ & $8800 \pm 170$ & $\sim 0.19$ & - & Hermes et al. $(2013)^{f}$ \\
\hline $\mathrm{J} 2228$ & $6.03 \pm 0.08$ & $7870 \pm 120$ & $\sim 0.16$ & - & Hermes et al. $(2013)^{f}$ \\
\hline
\end{tabular}

Notes. ${ }^{(a)}$ Nice, priv. comm. ${ }^{(b)}$ Based on Kaplan et al. (2013). See also Istrate et al. (2014) for further comments on the component masses of this source. ${ }^{(c)}$ The WD is most likely to have formed in this globular cluster binary given that the eccentricity is $e<10^{-5}$, as expected from recycling. ${ }^{(d)}$ Carter et al. (2011) found two possible solutions for $M_{\mathrm{WD}}\left(0.26 M_{\odot}\right.$ and $\left.0.37 M_{\odot}\right)$. This WD has $P_{\text {orb }}=3.3$ days and thus we adopt the lower value of $M_{\mathrm{WD}}$ since this is agrees much better with the known $\left(M_{\mathrm{WD}}, P_{\mathrm{orb}}\right)$-correlation (see Tauris $\&$ Savonije 1999, for discussions). ${ }^{(e)}$ See additional references therein. ${ }^{(f)}$ Pulsating He WDs, see Córsico \& Althaus (2014) for recent theoretical modelling.

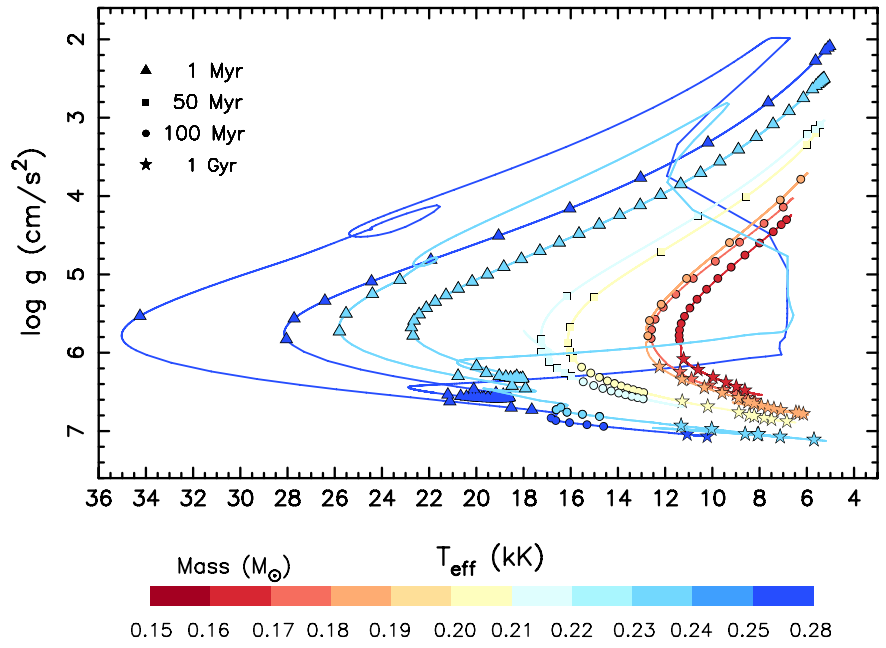

Fig. A.1. Selected tracks (Fig. 1) with a point marked for a time interval of $1 \mathrm{Myr}$ (triangle), $50 \mathrm{Myr}$ (square), $100 \mathrm{Myr}$ (circle), and $1 \mathrm{Gyr}$ (star).

In Fig. A.1 we have plotted points for fixed time intervals of evolution along a number of selected tracks from Fig. 1. The density of points along these curves combined with the (proto) WD luminosities at these epochs can be used to evaluate the probability of detecting them. For a direct comparison with data population synthesis needs to be included to probe the distribution of WD masses. The observational data plotted in Fig. 1 were taken partly from the sources given in Table A.1 (primarily He WDs with MSP companions, main-sequence A-star companions, or He WDs that have been detected to show pulsations).
Additional data for the plotted symbols can be found in Silvotti et al. (2012), Hermes et al. (2013), Brown et al. (2013).

\section{Appendix B: The (proto) WD contraction phase}

Figure C. 1 shows the time $\Delta t_{\text {proto }}$ it takes from Roche-lobe detachment until the proto-He WD reaches its highest value of $T_{\text {eff }}$ and settles on the cooling track. Shown in this plot are all our calculated models for progenitor stars of 1.2 and $1.4 M_{\odot}$ (i.e. a subset of the models plotted in Fig. 2). The black line (Eq. (1)) is an analytical result obtained from a somewhat steep core massluminosity function $\left(L \propto M_{\mathrm{WD}}^{7}\right)$ combined with the assumption (for simplicity) that in all cases $0.01 M_{\odot}$ of hydrogen is burned before reaching the highest $T_{\text {eff }}$. The figure shows that this line also serves as a good approximate fit to our calculated models. For a given He WD mass, the fit to $\Delta t_{\text {proto }}$ calculated from our models is accurate to within $50 \%$.

\section{Appendix C: Nuclear burning during flashes}

To compare the burning of residual envelope hydrogen for a case with and without large thermal instabilities (hydrogen shell flashes), we have plotted tracks in the HR-diagram shown in Fig. C.2. The age of the stars and the total amount of hydrogen remaining in their envelopes are given in Table C.1 for the points marked in the figure. These models were chosen very close to (but on each side of) $M_{\text {flash }} \simeq 0.21 M_{\odot}$, in both cases for a $1.3 M_{\odot}$ progenitor star. As discussed in the main text, although the peak luminosity is high during a flash (and thereby the rate at which hydrogen is burned), the star only spends a relatively short time $\left(\sim 10^{6} \mathrm{yr}\right)$ in this epoch. (For more massive He WDs it is even less time - for example, it only lasts $\sim 10^{3} \mathrm{yr}$ for a $0.27 M_{\odot}$ He WD.) Therefore, the amount of additional hydrogen 
A\&A 571, L3 (2014)

Table C.1. Ages and remaining hydrogen of the two proto-He WDs shown in Fig. C.2.

\begin{tabular}{rrrr}
\hline \hline Point & Relative age $^{a}$ & Total age $^{b}$ & $\begin{array}{r}\text { Hydrogen }^{c} \\
\left(10^{-3} M_{\odot}\right)\end{array}$ \\
\hline N1 & 0 & 0 & 13.68 \\
N2 & $341 \mathrm{Myr}$ & $341 \mathrm{Myr}$ & 2.94 \\
N3 & $1900 \mathrm{Myr}$ & $2240 \mathrm{Myr}$ & 0.79 \\
N4 & $8231 \mathrm{Myr}$ & $10470 \mathrm{Myr}$ & 0.67 \\
\hline F1 & 0 & 0 & 7.78 \\
F2 & $107 \mathrm{Myr}$ & $107 \mathrm{Myr}$ & 2.71 \\
F3 & $31 \mathrm{Myr}$ & $138 \mathrm{Myr}$ & 2.45 \\
F4 & $1536 \mathrm{yr}$ & $138 \mathrm{Myr}$ & 2.45 \\
F5 & $5.1 \mathrm{Myr}$ & $143 \mathrm{Myr}$ & 2.22 \\
F6 & $20 \mathrm{Myr}$ & $163 \mathrm{Myr}$ & 2.06 \\
F7 & $1536 \mathrm{yr}$ & $163 \mathrm{Myr}$ & 2.05 \\
F8 & $3.6 \mathrm{Myr}$ & $166 \mathrm{Myr}$ & 1.75 \\
F9 & $2089 \mathrm{Myr}$ & $2255 \mathrm{Myr}$ & 0.85 \\
\hline
\end{tabular}

Notes. The points N1...N4 are for the non-flashing $0.212 M_{\odot}$ WD model and the points F1...F9 are for the $0.221 M_{\odot}$ flashing WD model. ${ }^{(a)}$ Age relative to the previous point along the track. ${ }^{(b)}$ Cumulated age relative to the first point on the track (since the time of Roche-lobe detachment). (c) Total amount of hydrogen remaining in the envelope of the (proto)-He WD.

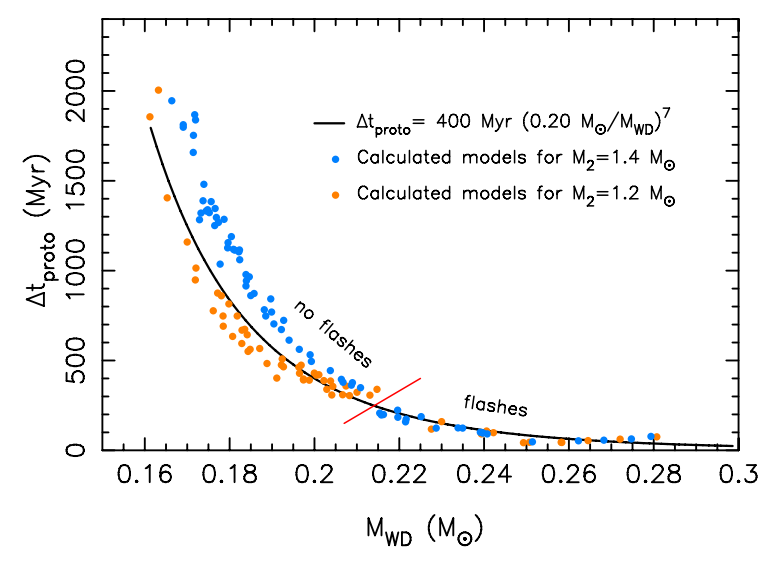

Fig. C.1. Calculated models of proto-He WDs from Fig. 2 for $M_{2}=$ $1.2 M_{\odot}$ (orange) and $M_{2}=1.4 M_{\odot}$ (blue). The black line is a fit to the data. It can also be derived analytically using a modified core massluminosity relation for low-mass evolved stars, combined with an assumed fixed amount of residual hydrogen $\left(0.01 M_{\odot}\right)$ to be burned. The red line separates models with and without flashes.

burned as a result of flashes is relatively small. In the example shown in Fig. C. 2 it amounts to about $12 \%$ of the total amount of hydrogen at the point of Roche-lobe detachment. Hence, the flashes may appear to reduce $\Delta t_{\text {proto }}$ by $\sim 100$ Myr. However, one must bear in mind that the proto-WDs that experience

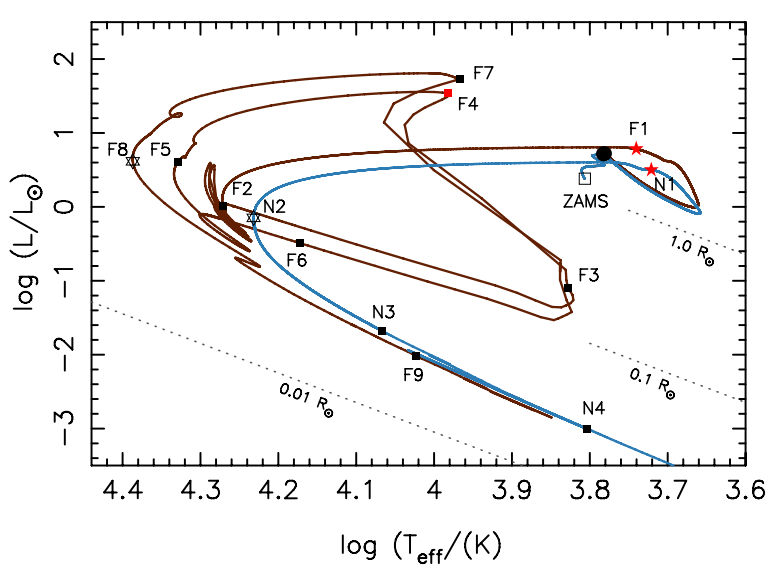

Fig. C.2. Evolutionary tracks in the HR-diagram for a $0.221 M_{\odot}$ protoHe WD with flashes (brown) and for a $0.212 M_{\odot}$ proto-He WD without flashes (blue). See Table C.1 for data.

flashes are also the WDs with the least amount of hydrogen in their envelopes after RLO.

For a star that experiences flashes, the residual hydrogen present in the envelope following the LMXB-phase is processed roughly as follows: $70 \%$ during the epoch from Roche-lobe detachment until reaching highest $T_{\text {eff }}, 10 \%$ during the flashes, and $20 \%$ after finally settling on the WD cooling track. 